\title{
Identification and Cross-Directional Control of Coating Processes: Theory and Experiments
}

\author{
Richard D. Braatz; Matthew L. Tyler, and Manfred Morari ${ }^{\dagger}$ \\ Chemical Engineering 210-41, California Institute of Technology, Pasadena, CA 91125 \\ Ferdinand R. Pranckh and Luigi Sartor \\ Avery Research Center, 2900 Bradley St., Pasadena, CA 91107-1599
}

\begin{abstract}
Abetract
Of special industrial interest is the crosedirectional control of continuous coating processes, where the cross-direction refers to the direction perpendicular to the substrate movement. The objective of the controller is to maintain a uniform coating under unmessured process disturbances based on assumptions relevant to coating pro cesses found in industry. A model for control design is developed. This model is used to derive a model predictive controller with the objective of maintaining flat profiles of coating across the qubatrate by varying the liquid fows along the croes direction. Actuator constraints, measurement noise, and model uncertainty are investigated to determine which of these limit the achievable cloeed loop performance. From a knowledge of the effect of these limitations on performance we determine how the plant could be modified to improve the costing uniformity. The theory developed throughout the paper is rigorously verified though experiments on an industrial pilot plant.
\end{abstract}

\section{Introduction}

Coating refers to the coating of a substrate with a uniform layer of liquid. Coating processes are of great importance to manufacturing, especially in the photographic, magnetic and optical memory, electronic, adhesive, and paper industries.

Plant Description Fig. 1 is a simplified diagram of a typical plant. The process begins with a feed roller from which substrate is unwound. From there, the substrate passes between a roller and a stainles steel die. The liquid flows through a slot in the die to the substrate. The cavity in the die is designed to distribute a uniform flow of liquid through the slot. A controlled pump supplies a constant flow of liquid through the die.

The term "gap width" refers to the distance across the slot at a given point along the die. The gaps through which liquid fows are adjusted by means of $n$ equally spaced bolts. The bolts are adjusted manually.

After being coated with liquid, the substrate passes through a drier. After the drier, the time-averaged coating thickness at each of the $n$ positions corresponding to the die bolts is measured by a traversing coat-weight sensor. The coated substrate is wound on the product roller.

Control Objective The crose-directional control problem is aimed at maintaining a uniform profile of liquid across the substrate. Suc cessful control of coating thickness improves product quality and reduces the time needed to bring the plant on-line. Poor control can lead not only to coating thictness nonuniformity but also coating instabilities that leave portions of the substrate uncovered; such substrate must be rejected (for a short summary of coating instabilities, see Sartor, 1990).

We will consider coating processes with a large time delay between a change in gap width and the resulting sensing of the change in coating profile downstream. This time delay could be due to a sensor installed at a fair distance from the die as in the coating plant considered above. Because the controller cannot be expected to reject disturbances faster than this time delay, detailed proces dynamics are not considered in the modeling, identification, and control of the cross-directional coating process. Thus the objective of the controller is the elimination of slow disturbances in the coating thickness. The disturbances were of this nature in the Avery/Dennison

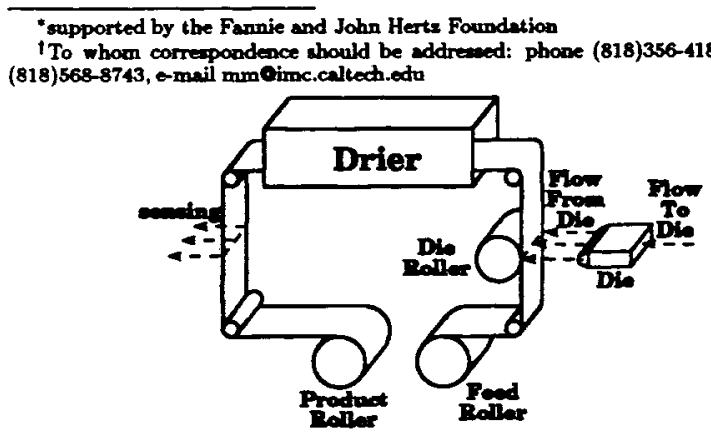

Figure 1: Typical coating plant pilot plant; the control of this plant is atudied in this subetrate. Organisation Arsumptions that are relevant to a subset of coating processes found in industry are used to develop a model for control design. This model is used to derive an unconstrained model predictive controller to maintain flat profiles of liquid acroes the substrate by varying the gap widths. The unconstrained controller is modified to prevent physically infeasible actuator movements (gap widths).

Actuatos constraints, measurement noise, and model uncertainty are investirated to determine which of these limit the achievable closed loop performance. The theory developed throughout the paper is applied to a pilot plant liquid coating process at the $\mathrm{Av}$ ery/Dennison Research Center in Pasadena.

Notation All scalars are italicized. Matrices are upper case bold. $M_{i, j}$ represents the $(i, j)$ element of the matrix $M$. Vectors are lower case and bold. The ith element of the vector $x$ is represented as $x_{i}$. $x(t)$ refers to the value of $x$ at time $t$

\section{Model Development}

Below we make assumptions on the plant that are relevant to a subset of coating proceseses found in industry. These assumptions are used to develop a dimensional model. This model is transformed to a dimensionless form. The dimensionless model is then rearranged into a form suitable for controller deaign.

2.1 Dimensional Model

Consider a plant with the number of actuators $n$ equal to the number of sensors (or sensor measurement positions). It can be shown theoretically and experimentally (through examination of pilot plant data) that the plant behaves approximately linearly in the operating region. Let $u$ be the vector of gap widths, $z$ be the vector of coating thickneses, and collect any effects on the coating thickness not due to changes in gap width. If the process dynamics are approximated by a pure delay, then the coating thickness at sampling instant $t$ is related to the gap width at the previous sampling instant through

$$
\mathbf{x}(t)=\mathbf{P} \breve{u}(t-1)+\forall(t),
$$

where $P$ is a constant $n \times n$ matrix.

Assumption on $\forall$ accounts for unmeasured input effects such as measurement noise and disturbances. We assume that $\breve{v}$ is a non-zero-mean stochastic variable, i.e. $\{\checkmark(0), \forall(1), \ldots, \forall(h), \ldots\}$ is a sequence of independent random vectors with non-zero mean [6]. We define the steady-state disturbance $\breve{d}$ as the time-averaged value of $\breve{v}$, and define $\breve{n}$ by $\mathbf{x}(t)=\breve{v}(t)-\breve{d}$. We will assume that $\breve{n}$ is white noise. It will be referred to as measurement noise.

$\checkmark$ is chosen to be stochastic because it describes well the apparently random fluctuations of the process. In practice, equal gap widths do not give a uniform coating because of imperfections in the roller or the die, non-uniformities in the drying process, or poor calibration of the gap widths. These imperfections lead $\vec{v}$ to have non-zero mean.

Assumptions on P Typically, the total flow of coating through the die is maintained constant through a high gain controller. Because of constant total flow, increasing the flow through one actuator will necessitate decreasing the flow through the others. In the development of the model, we make the following assumptions:

1.The total liquid flow (and therefore the sum of the coating thicknesees) is constant.

2. All actuators and sensors are equivalent.

3. The only interactions between the actuators are due to the constant flow aseumption.

Assumption 2 implies that $\mathbf{P}$ is symmetric. Assumption 3 implies that $\mathbf{P}$ can be separated into two matrices

$$
\mathbf{P}=\breve{k} \mathbf{I}-\mathbf{M} \text {, }
$$

where $K$ is the gain between the ith gap width and its corresponding coating thicknes for an infinitely wide die (i.e. $n \rightarrow \infty)$. I is the $n \times n$ identity matrix, $\not K I$ is the contribution that changing gap widths would have on the coating thicknesece if there were no interactions, and $M$ represents the effect that increasing one gap width has on decreasing the fiom through all the gaps. Assumption 3 also implies that all elements of $M$ are equal, i.e. $M_{i, j}=m$ for $i, j=1,2, \cdots, n$. 
Then

$$
\mathbf{P}=\underbrace{\left(\begin{array}{ccccc}
\check{k}-m & -m & -m & \ldots & -m \\
-m & \check{k}-m & -m & \ddots & \vdots \\
-m & \ddots & \ddots & \ddots & -m \\
\vdots & \ddots & \ddots & \check{k}-m & -m \\
-m & \cdots & -m & -m & \check{k}-m
\end{array}\right)}_{n \times n}
$$

Assumption 1 implies that $\sum_{i=1}^{n} \underline{x}_{i}$ is constant for all gap widths Then (ignoring the noise $\overline{\text { n), }}$, we have from (1) that

$$
\sum_{i=1}^{n} \check{x}_{i}(t)=\sum_{i=1}^{n}\left(\breve{d}_{i}+\sum_{j=1}^{n} P_{i, j} \breve{u}_{j}(t-1)\right)=\sum_{i=1}^{n} \breve{d}_{i}+\sum_{j=1}^{n}\left(\sum_{i=1}^{n} P_{i, j}\right) \breve{u}_{j}(t-1)
$$

must be a constant for all $\check{u}_{j}(t-1)$. This implies that

$$
\sum_{i=1}^{n} P_{i, j}=0, \quad \text { for } j=1,2, \cdots, n \text {. }
$$

By substituting the elements of $P$ from (3) into the summation (5), we find that $m$ must be related to $k$ by

$$
m=\check{k} / n \text {. }
$$

Substituting for $m$ in (3) gives the final form for $P$ :

$$
\mathbf{P}=(\check{k} / n) \mathbf{B},
$$

where

$$
\mathbf{B}=\underbrace{\left(\begin{array}{ccccc}
n-1 & -1 & -1 & \cdots & -1 \\
-1 & n-1 & -1 & \ddots & \vdots \\
-1 & \ddots & \ddots & \ddots & -1 \\
\vdots & \ddots & \ddots & n-1 & -1 \\
-1 & \cdots & -1 & -1 & n-1
\end{array}\right)}_{n \times n} .
$$

The single model parameter $\breve{k}$ does not depend on the number of actuators $n$.

\subsection{Dimensionless Model}

The model is transformed to a dimensionless form for two reasons. First, using a dimensionless model will allow the control parameters to vary little between different plants. Second, the controller is designed to produce a costing of uniform thickness and will not be able to change the mean coating thickness. A flow controller which maintains constant flow to the coating die is used to adjust the mean coating thickness. Therefore the non-dimensional variable $\boldsymbol{x}$ is chosen to represent coating thickness as a deviation from the mean.

Define $\bar{x}=\frac{1}{n} \sum_{i=1}^{n} \breve{x}_{i}$ and $\bar{u}$ as the nominal gap width. The nominal gap width should be chosen well within the stable coating region. Define the following dimensionless variables:

$$
x_{i}=\frac{\breve{x}_{i}-\bar{x}}{\bar{x}}, \quad u_{i}=\frac{\check{u}_{i}-\bar{u}}{\bar{u}}, \quad d_{i}=\frac{\breve{d}_{i}-\bar{x}}{\bar{x}}, \quad n_{i}=\frac{\check{n}_{i}}{\bar{x}}, \quad k=\frac{\check{k}_{\bar{u}}}{n \bar{x}}
$$

Solve the above expressions for $\breve{z}_{i}, \breve{u}_{i}, \breve{d}_{i}, \breve{n}_{i}$, and $\breve{k}_{\text {, substitute }}$ into (1), and rearrange to give the dimensionless model:

$$
\mathbf{x}(t)=k B \mathbf{u}(t-1)+d+\mathbf{n}(t)
$$

2.3 Model for Control Design

The matrix $B$ in (8) is singular. This is because the coating thicknesses $x$ are not uniquely determined by the gap widths $u$. Any increment in gap width added to all the gap widths $u_{i}$ does not change the coating thicknesses. However, to keep a stable film, the dimensionless gap widths u must not stray too far from the preferred position of $\mathbf{0}$. We augment the model with the additional equation $n u_{i}=0$ to both keep $u$ from straying and to give a unique mapping of the coating thicknesses to the gap widths. This is done as follows:

- Add a component to $x$, $d$, and $n$, and set this component to zero, i.e. $x_{n+1}=n_{n+1}=d_{n+1}=0$

-Add a row of ones to the plant matrix $k B$ to give the new $(n+1) \times n$ plant matrix $C=\left[\begin{array}{c}k B \\ 1 \cdots 1\end{array}\right]$.

This leads to the augmented model

$$
\mathbf{x}(t)=\mathbf{C u}(t-1)+\mathbf{d}+\mathbf{n}(t) .
$$

Since the mean value of $u$ is a free independent variable (it does not change coating thicknesses), a controller design based on the above model which seeks to minimize $x$ will automatically adjust its control action so that the mean value of $u$ will be exactly zero. Also, the singularity of the original gain matrix $\mathbf{B}$ is removed; $\mathbf{C}$ has full column rank.
To derive the model predictive controller in the next section, it is convenjent to express the model in terms of the changes in the inputs rather than the inputs themselves. For this purpose, we subtract the equation (11) for $t-1$ from that at $t$ to arrive at

$$
\boldsymbol{x}(t)=\mathbf{x}(t-1)+\mathbf{C} \Delta \mathbf{u}(t-1)+\Delta \mathbf{n}(t),
$$

where

$$
\Delta \mathbf{u}(t-1)=\mathbf{u}(t-1)-\mathbf{u}(t-2)
$$

The controller calculate the inputs to the plant based on the measured variables. The model for control design is:

$$
\begin{aligned}
& x(t)=x(t-1)+C \Delta u(t-1) \\
& 3 \quad \text { Eatimation and Prediction }
\end{aligned}
$$

\section{Eatimation and Prediction}

Recall that our objective for using a model is to predict the effect of changes in gap widths on the conting thicknesses. This will allow us to find the "beat" adjustments in gap widths to reject disturbances.

3.1 State Estimation - Filter

The state estimator is most conveniently expressed in the following two-step form [3]:

Model Prediction:

$$
x(t \mid t-1)=x(t-1 \mid t-1)+\mathbf{C} \Delta \mathbf{u}(t-1) .
$$

Correction Besed on Measurements:

$x(t \mid t)=x(t \mid t-1)+\gamma[\hat{x}(t)-x(t \mid t-1)]$,

$x(\cdot \mid t-1)$ denotes the estimate of $x(\cdot)$ based on measurements up to time $t-1$. $\hat{x}(t)$ is the measurement of $x$ at time $t . \gamma \in(0,1)$ is a filter parameter used to filter noise and to obtain robustness to model uncertainty. The larger the measurement noise and model uncertainty, the smaller $\gamma$ should be chosen.

By substituting (15) into (16) we obtain the state estimator

$\mathbf{x}(t \mid t)=(1-\gamma)[\mathbf{x}(t-1 \mid t-1)+\mathbf{C} \Delta \mathbf{u}(t-1)]+\gamma \hat{\mathbf{x}}(t)$ which allows one to compute the current state estimate $x(t \mid t)$ based on the previous estimate $x(t-1 \mid t-1)$, the previous input move $\Delta \mathbf{u}(t-1)$, and the current measurement $\dot{\mathbf{x}}(t)$. The state estimator is initialized with $x(0 \mid 0)=\dot{x}(0)$.

The state estimator (17) suggests that $x(t \mid t)$ is a filtered version of $\hat{\boldsymbol{x}}$. Indeed, in a noise-free system with the manipulated variables constant, we have

$$
x(t \mid t)=(1-\gamma) x(t-1 \mid t-1)+\gamma \hat{x}(t),
$$

which shows that the state estimate $x(t \mid t)$ is $\hat{x}$ passed through a first order filter. If the output $\hat{x}$ suddenly changes to a constant value then the state estimate $x(t \mid t)$ approaches the true value $\hat{x}$ with the filter time constant:

$$
\tau=-T_{s} / \log (1-\gamma)
$$

where $T$, is the time between sampling instances [3]

3.2 Prediction

The control algorithm prescribes the gap widths $u$ which reject disturbances in $x$. In order for the control algorithm to determine the "best" current gap widths there has to be a means for predicting the effect of the gap widths on the future coating thicknesses $x$. The predictor is given by writing (15) for the next time step $t+1$

$$
x(t+1 \mid t)=x(t \mid t)+C \Delta u(t)
$$

$$
4 \text { Control }
$$

We begin by stating the unconstrained control objective. We derive the unconstrained controller that minimizes this objective. Then we discuss three methods of modifying this controller to handle actuator constraints, in our case constraints in adjacent gap widths. 4.1 Unconstrained Control Algorithm

Performance Criterion The performance criterion is to minimize the quadratic objective

$$
z=\|x(t+1 \mid t)\|^{2}
$$

where $\|\cdot\|$ represents the Euclidean norm, $\|x\|^{2}=\sum_{i=1}^{n} x_{i}^{2}$.

Unconstrained Control Problem We express the control problem as an optimization by combining the objective (21) with the predictor (20):

$$
\min _{\Delta \mathbf{u}(t)}\|\mathbf{x}(t+1 \mid t)\|^{2}, \quad \text { where } \mathbf{x}(t+1 \mid t)=\mathbf{x}(t \mid t)+\mathrm{C} \Delta \mathbf{u}(t) \text {. }
$$

The least-squares solution to the unconstrained control problem is

$$
\Delta \mathbf{u}(t)=-\left(\mathbf{C}^{T} \mathbf{C}\right)^{-1} \mathbf{C}^{T} \mathbf{x}(t \mid t) .
$$

4.2 Handling Actuator Constraints

Excessive streses in the die constrain adjacent actuator positions. The specification is that the difference between adjacent actuator positions is limited, i.e.

$\left|\delta u_{i}\right|=\left|u_{i+1}-u_{i}\right| \leq \mid \delta u_{\text {mas }}$

for $i=1, \ldots, n-1$

Constraint-handling will be needed when the disturbances are sufficiently large and have sherp spatial variations acmes the substrate. When the disturbances are uniform across the substrate, then the control action calculated from the unconstrained control ajgorithm will be uniform, and constraint-handling is not needed.

Actuator constraints can be handled in three ways: 1) by including additional terms in the objective function, 2) by adding 
the constraints explicitly to the control algorithm, or 3) by scaling the control actions to be "feasible", i.e. to satisfy the constraints. These constraint-handling methods are compared is detail in [4] The third constraint-handling method was choeen. The first method was ruled out because additional weighted terms in the objective function always affect the control action, giving poorer performance when constraint-handling is not needed. The second method was ruled out because it is not as simple to implement and analyze as the third method, and because it was shown in simulations that for the Avery/Dennison pilot plent in Section 5.9 the third method performs nearly as well the second method. The scaling method is easy to implement and performs exactly as the unconstrained algorithm when constraint handling is not needed.

Scaling Control Actions Constraints can be handled by projecting any infeasible $u$ given by the unconstrained control law (23) to the feasible space. Fig. 2 illustrates this idea for the first constraint (24) for $n=3$. All feasible control actions u are given by the shaded region. When the unconstrained control law (23) suggests an infeasible control action, a feasible control action is found by projecting $\mathbf{u}$ to the feasible space. Many projections could be used, but the projection shown (which involves simple scaling of the control action) maintains the direction of the control action, which can be important for multivariable systems [2]

Now consider satisfying the first constraint (24) for general $n$. This is done by scaling the control action $u$ calculated from the unconstrajned control law (23):

$$
\mathbf{u}^{\dagger}(t)=\left\{\begin{array}{cl}
\mathbf{u}(t) & \max _{i}\left|\delta u_{i}(t)\right| \leq|\delta u|_{\text {max }} \\
\frac{|\delta u| \max _{i}}{\max _{i}\left|\delta u_{i}(t)\right|} \mathbf{u}(t) & \max _{i}\left|\delta u_{i}(t)\right|>|\delta u|_{\text {max }} .
\end{array}\right.
$$

$\mathbf{u}^{\dagger}$ satisfies the constraint (24).

Constrained Control Algorithm In summary, the constrained control algorithm is:

- Calculate the estimated state through (17).

- Calculate the unconstrained control move from (23).

- Scale the unconstrained control move using (25) to obtain the constrained control move which is implemented. The state estimator for the next step (17) will use the constrained implemented move from the previous atep.

\section{Limits of Performance}

We would like to know how well the controller can be expected to reject disturbances in coating thicknesses. This leads us to study the various factors that limit the achievable closed loop performance. Knowledge of how these limitations affect the performance can show us how to modify the plant to improve the uniformity of the coating process. Also, because identification of model parameters is timeconsuming and costly, we study how accurate the identification must be to achieve a given level of performance. We would also like to compare the performance of our control algorithm to the best closed loop performance achievable by any control algorithm. This allows us to convince ourselves that we have indeed designed the best possible controller.

We begin by making the assumptions necessary to achieve perfect one-step rejection of disturbances. This provides a standard to which the various limitations on the closed loop performance can be compared.

Perfect Control We are interested in the ability of the controller to reject slow disturbances. Let us study the rejection of a steadystate disturbance and let the control algorithm start at $t=0$. For simplicity of presentation, let the disturbance $d$ have zero-mean and the initial gap widths $u(-1)=0$. If we make the following three assumptions:

1. no actuator constraints,

2.no measurement noise, and

3.our model is exactly equal to our plant

then it can be shown that the control algorithm with $\gamma=1$ perfectly rejects the steady-state disturbance in one step.

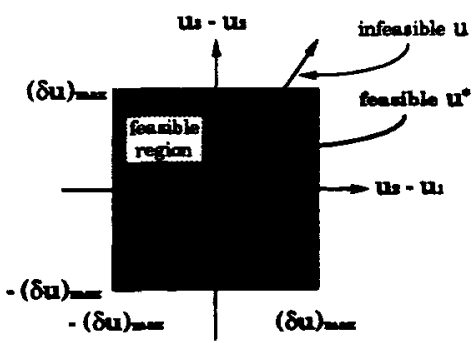

Figure 2: Projection of an infeasible control action to the feasible space.
We will drop the assumptions of no actuator constraints, no measurement noise, and no model uncertainty in turn and show how each of these prevent the controller from rejecting the steady-state disturbance in one step.

\subsection{Constraints on Actuator Movements}

The constraints on the actuator positions will degrade performance only when the the control move from the unconstrained algorithm must be scaled to keep the gap widths feasible. It can be shown that in this case the costing thicknesses at the next time $x(1)$ do not equal zero. We will also show below that the coating thicknesses $x$ may never reach zero.

Assurne no measurement noise, $\gamma=1$, that the model is perfect, and for simplicity of presentation that $\mathbf{d}$ has zero mean and the initial gap widths $\mathbf{u}(-1)=0$. Then the measured costing thickneses at $t=0$ is $\hat{\mathbf{x}}(0)=\mathbf{x}(0)=\mathrm{d}$. The control move for the first step from (23) is

$$
\mathbf{u}(0)=-\left(\mathbf{C}^{T} \mathbf{C}\right)^{-1} \mathbf{C}^{T} \mathrm{~d} .
$$

If the control move from the unconstrained algorithm must be scaled to keep the gap widths feasible, the constrained control move is

$$
\mathbf{u}^{\dagger}(0)=-\lambda\left(\mathbf{C}^{T} \mathbf{C}\right)^{-1} \mathbf{C}^{T} \mathrm{~d},
$$

where $0<\lambda<1$. If the operator implements the control move $\mathbf{u}^{\dagger}(0$ exactly and there is no measurement noise, then applying the contro move to the plant (11) gives that (after some matrix manipulation)

$$
x(1)=(1-\lambda) d \text {. }
$$

We see that the effect of the disturbance has been diminished by factor of $1-\lambda$. It can be shown that under the given assumptions the control move will not change, and the coating thicknesses will continue to $x(t)=x(1)=(1-\lambda) d$

The constraints on gap widths prevent the steady-state disturbance from being completely rejected. This is true regardless of the control algorithm used.

Plant Modifications to Improve Performance The gap widths are constrained to prevent bigh stresees in the die. A die can be designed to have weaker constraints on its die gap widths by either placing the bolts further apart, by making the die lip thinner, or by making the die out of a more flexible metal. Putting the die bolts too far apart leads to strips of uncontrolled coating thickness between the die bolts. Machining a die to tight tolerances becomes increasingly difficult as the die metal becomes thinner or more flexible.

5.2 Measurement Noise

Measurement noise al ways limits performance. A noise filter is used to diminish the effects of noise. Because increased noise filtering also slows the controller response time, there is a tradeoff between improved coating uniformity and slower response times. We now define a measure of coating uniformity and study this tradeoff in more detail.

Consider the closed loop system with a perfect model without disturbances, only measurement noise. For a stabilizing controller, the expected value for the estimated state $x(t \mid t)$ is zero. The estimated state will not exactly equal zero because the controller will treat the measurement noise as a disturbance and will try to reject it. Thus the estimated state will have some variance depending on the size of the noise. The variance of the estimated state $x(t \mid t)$ is an appropriate measure of the uniformity of the coating. For simplicity of presentation, assume a perfect model and that the noise at each gap position is equal--dropping these assumptions only slightly affects the following. Then it can be shown that

Variance $\left(x_{i}\right)=(\gamma /(2-\gamma))$ Variance $\left(n_{i}\right) \quad$ for $i=1, \ldots, n .(29)$

A measure of the controller's speed of response is the filter time constant plus 1 , i.e. $\tau+1$ (The ' 1 ' accounts for the delay through the plant).

Both Variance $\left(x_{i}\right)$ and $\tau$ (through (19)) are functions of the noise filter parameter $\gamma$. Fig. 3 compares the controller response time versus the ratio of the variance of the state estimate to the measurement noise for different values of $\gamma$. A small amount of filtering $(\gamma \rightarrow 1)$ corresponds to fast response times, but poor coating uniformity. A large amount of filtering corresponds to good coating uniformity, but with slow response times.

Plant Modifications to Improve Performance Ways to decrease the sensor noise should be investigated. The cables to the sensor should be shielded adequately to keep the sensor noise as small as possible. The effect of air currents can be diminished by decreasing the distance between the sensor and the coated substrate. The vibration of the substrate and the sensor should be minimized. Of course, an accurate sensor reading requires a stable film.

5.3 Model Uncertainty

Model uncertainty is the difference between the model and the plant. The error between the true behavior of the physical process and that predicted by the model can significantly affect the ability of the control system to perform adequately. Controllers that are insensitive to model uncertainty are said to be robust. Below we quantify the effeet of uncertainty. More specifically, we show that the control algorithm 


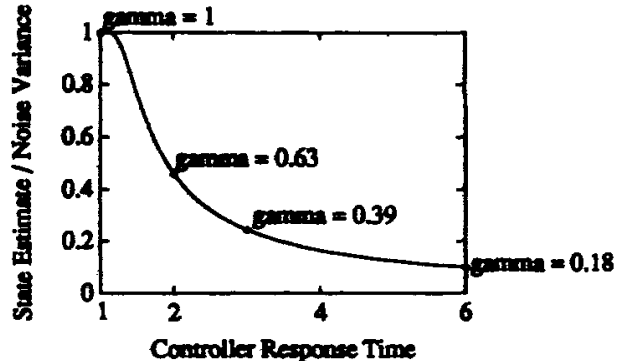

Figure 3: Comparieon of variance of state estimate to measurement noise ratio versas controller response time.

proposed in this substrate is robust to gain uncertainty. Also, we will analyze the robugtnes as a function of the filter parameter $\gamma$ to determine the effect of the noive filter on robustness.

Uncertainty in Gain Matrix The closed loop stability can be analyzed from the state-space equation for the closed loop system. A system will be considered stable when the effect of small disturbances remains small. A system is considered unstable when the effect of small disturbances grows until the constraint (24) is reached. The effect of disturbances will never grow unbounded because (24) and $\sum_{i=1}^{n} u_{i}=0$ are satisfied, which bound the magnitude of the control action.

Let the measurement be described in terms of the real plant: $\hat{\mathbf{x}}(t)=\mathbf{C}_{\mathbf{r}} \mathbf{u}(t-1)+\mathbf{v}_{\mathbf{r}}(t)$.

No assumptions are made on the unmeasured inputs $v_{r}$.

Define $\Gamma$ by $\Gamma=-\left(\mathbf{C}^{T} \mathbf{C}\right)^{-1} \mathbf{C}^{T}$. Then the control law (23) is given by

$$
\mathbf{u}(t)=\mathbf{u}(t-1)+\mathbf{r} \mathbf{x}(t \mid t)
$$

Substitute $x(t \mid t-1), \hat{x}(t)$, and $u(t-2)$ from (15), (30), and (31) into (17) and rearrange to give

$x(t \mid t)=(1-\gamma)(I+C r) x(t-1 \mid t-1)+\gamma C_{\mathbf{r}} u(t-1)+\gamma v_{\mathbf{r}}(t)$ Substitute $x(t \mid t)$ from (32) into (31) to give

$\mathbf{u}(t)=(1-\gamma) \Gamma(I+C \Gamma) x(t-1 \mid t-1)+\left(I+\gamma \Gamma C_{\mathbf{r}}\right) \mathbf{u}(t-1)+\gamma \Gamma \mathbf{v}_{\mathbf{r}}(t)$

Let $u(t)$ be a state, then (32) and (33) give the state-space equation that defines the closed loop system,

$\left[\begin{array}{c}x(t \mid t) \\ \mathbf{u}(t)\end{array}\right]=\left[\begin{array}{cc}(1-\gamma)(\mathbf{I}+\mathbf{C r}) & \gamma \mathbf{C}_{\mathbf{r}} \\ (1-\gamma) \Gamma(I+C \Gamma) I+\gamma \Gamma C_{\mathbf{r}}\end{array}\right]\left[\begin{array}{c}x(t-1 \mid t-1) \\ \mathbf{u}(t-1)\end{array}\right]+\left[\begin{array}{c}\gamma \\ \gamma \Gamma\end{array}\right] v_{\mathbf{r}}(t)$.

For a discrete time system, we have closed loop stability if and only if the eigenvalues of

$$
A=\left[\begin{array}{cc}
(1-\gamma)(I+C \Gamma) & \gamma C_{\mathbf{r}} \\
(1-\gamma) \Gamma(I+C \Gamma) & I+\gamma \Gamma C_{\mathbf{r}}
\end{array}\right]
$$

are inside the unit circle. More specifically, the effect of disturbances will decay to sero if the spectral radius of $A$ is less than one, and the effect of small disturbances will grow until the constraints are met when the spectral radius of $A$ is greater than one [1].

Uncertainty in Gain This rection considers uncertainty in the gain; interaction uncertainty for the Avery/Dennison pilot plant will be considered in Section 6.2. The real plant gain will be denoted as $k_{r}$ and the augmented real plant is $C_{r}=\left[\begin{array}{c}k_{r} B \\ 1 \cdots 1\end{array}\right]$. Recall that $k$ is the gain and $\mathbf{C}$ is the gain matrix for the model.

By calculating the eigenvalues of $A$ in (35) we determine which values of the ratio $K=k / k$, give a stable closed loop system for each value of filter parameter $\gamma$ (se Fig. 4). If the gain of the real plant is not underestimated by more than a factor of two $(K>1 / 2)$, then the cloeed loop system is stable. For increased filtering (smaller $\gamma$ ) the model gain $k$ need not be as accurate. In other words, increased filtering adds robustness to gain uncertainty. It can be shown that the stability boundary in Fig. 4 is the straight line given by $k=$ $\gamma \boldsymbol{k}_{\mathbf{p}} / 2$.

The plant gain need not be known accurately for the controller to remain stable. Uncertainty in the plant gain will lead only to slower rejection of disturbances. Since we need approximate only a plant gain to deaign the controller, detailed identification runs are unnecesary for controller design. Any ressonable estimate will do. This makes it easier to apply the control algorithm to new crossdirectional systems when $\tilde{k}$ does not change much between systems.

It is well-known that high condition number plants (called illconditioned may be sensitive to actuator uncertainty [5]. It is shown in [4] that that ill-conditioning is not a serious problem for crosedirectional processes of the type studied here.

6 Application to Avery/Dennison Pilot Coater

The theory developed in the preceding section is applied to the control of a pilot plant coater at A very/Dennison Research Center

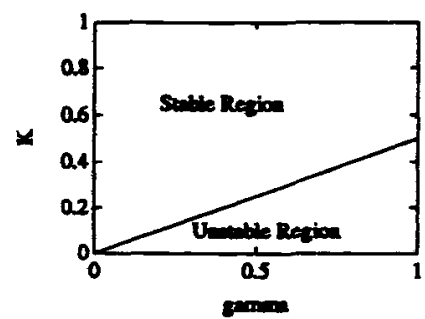

Figure 4: Clooed loop atability a function of $\gamma$ and $K=k / k_{r}$, no interaction uncertainty.

\begin{tabular}{|l|r|}
\hline die width & $0.35-2.5 \mathrm{~m}$ \\
\hline die bolk spacing & $30-60 \mathrm{~mm}$ \\
\hline coating thicknes & $10-60 \mathrm{~mm}$ \\
\hline coating weight & $15-50 \mathrm{~g} / \mathrm{m}^{2}$ \\
\hline substrate speed & $0.5-6 \mathrm{~m} / \mathrm{s}$ \\
\hline
\end{tabular}

Table 1: Typical ranges of physical parameters for adhesive coaters

(see Fig. 1). Typical ranges of physical parameters for such coaters are given in Table 1 .

First the model is identified and the model aesumptions are jus tified based on input-output data. Then the effect of interaction uncertainty on the stability of the closed loop system was investigated using the model fit to the pilot plant data. This was done to ensure that uncertainty in the interactions (i.e. deviations from the structure implied by (3)) would not cause the controller to perform poorly. We then demonstrate that the controller can be effectively tuned on-line. We conclude the section with an experimental closed loop test of the controller.

6.1 Identification

For the pilot plant, the number of actuators $n=12 . k$ was fitted by least-aquares from fifty input-output data sets. In Fig. 5 the predicted coating thicknesecs are compared with experimental dat for a typical input.

To test the anmptions used to develop the form of the gain matrix $P$ in Section 2.1 , we fitted the entire $12 \times 12$ gain matrix in (1) to estimate a total of 144 parameters-we denote this matrix by $\mathbf{P}_{144}$. As shown in Fig. 5, this model gives little improvement over the gain matrix $\mathbf{P}$ satisfying the assumptions, so the assumptions on $P$ are valid.

The die had been designed to give a small interaction between nearest-neighbor positions. Assumption 3 in Section 2.1 would not have been justified if the spacing between the actuators had been much smaller.

6.2 Robustness to Interaction Uncertainty

The effect of interaction uncertainty on the stability of the closed loop system was investigated using the model fit to the pilot plant dats. This was done to ensure that uncertainty in the interactions would not cause the controller to perform poorly. The same procedure as in Section 5.3 was used, but with $\mathbf{C}_{\mathbf{r}}=\left(\begin{array}{c}\mathbf{P}_{144} \\ 1 \cdots 1\end{array}\right)$ for the real plant and $\mathrm{C}=\left(\begin{array}{c}k \mathrm{~B} \\ 1 \cdots 1\end{array}\right)$ for the model. Fig. 6 shows the stable region as a function of the normalized model gain $K=k / k_{r}$ where $k_{r}$ denotes the beat fit gain. As in Fig. 4 , the boundary between the stable and unstable regions is a straight line, but the slope in Fig. 6 is steeper. Introducing interaction uncertainty decreases the stable region, but an accurate estimate of $k$ is still not required. This will be experimentally verified below.

6.3 Experimental Closed Loop Control

The main purpoee of the experiments was to verify that detailed identification of $k$ is not required for the resulting controller to give good performance. This is important because gathering detailed

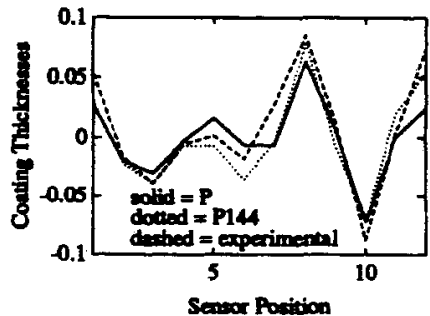

Figure 5: Comparison of coating thicknesses predicted by $\mathbf{P}$ and $\mathbf{P}_{144}$ with experimental data. 


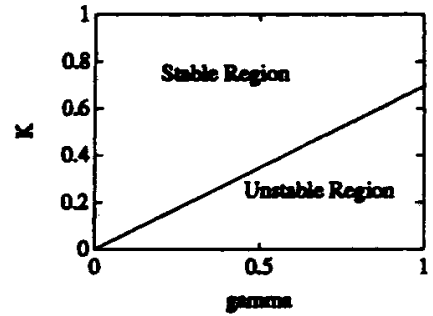

Figure 6: Cloeed loop stability as a funetion of $\gamma$ and $K=k / k$. Interaction uncertainty was included throngh the use of $\mathbf{P}_{144}$.

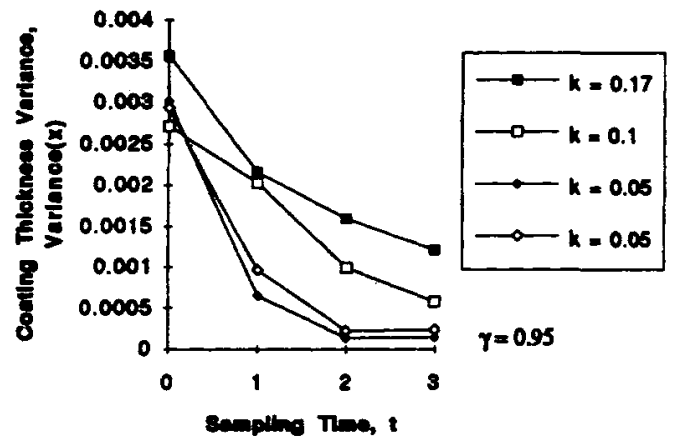

Figure 7: Comparison of coating thickneas variances. The control actions calculated using $k=0.025$ were excessively large and were not implemented.

input-output data is expensive.

All the die gaps were set equal to their nominal value. Because of imperfections in the die and roller and inaccuracy in the die gap settings, this gives non-uniform costing thicknesses. The goal of the controller is to make the coating thicknesses uniform. This disturbance is small enough that constraint-handling was not needed. Because the number of experiments was limited, we deeided to perform all experiments with a fixed $y$ near one. As discussed in Section 5.2, in plant operation $\gamma$ would be chooen to trade off the closed loop speed of response with the variance of the coating thictneses.

There were two major differences between the coater used for the identification experiments and the coater used for the closed loop experiments. First, the measurement noise was smaller for the second coater. Second, the coaters had different dies, so the responses with the two dies are expected to be different. A comparison of the die designs showed that the interactions are neglizible for both dies but the steady state gains $k$ are expected to be subotantially different. Because experiments are costly, our strategy was to avoid reidentifying $k$ from open loop experiments but to perform closed loop experiments instead for a few values of $k$ and chooee the one that gives good control-effectively determining the optimal $k$ through on-line tuning.

Fig. 7 shows the variance of the coating thicknesses for $k=$ $0.17,0.1$, and 0.05 . Since $\gamma$ was chooen near 1 and the interactions were negligible, we expect a fast response when the model steady state $\operatorname{man} k$ is close to the true gain. Because the gain $k=0.17$ identified for the previous die gave slow response, the controller gain is too small. This implies that the steady state gain for the model is too large. The response for $k=0.1$ also gave sluggish response. Therefore we tried a smaller $k$. For $k=0.05$, the disturbance was rejected in two sampling times.

If we had perfect control and $\gamma=1$, the disturbance would be rejected in one sampling time. If the assumptions of perfect control in Section 4.2 were satisfied with $\gamma=0.95$ then the closed loop time constant would be $\tau+1=1 / \log (1 /(1-0.95))+1 \approx 4 / 3>1$. Since we do not satisfy all the assumptions of perfect control, we cannot expect the disturbance to be rejected is less than two sampling times, i.e. $k=0.05$ gives the best achievable performance. We see that $k$ needed to design the controller was determined from only three closed loop experiments.

From Fig. 6 we expect that using $k$ much less than 0.05 would give poor performance. This agrees with experiment-the control actions calculated using $k=0.025$ were excessively large and were not implemented.

Fig. 8 shows the closed loop response for $k=0.05$. The dis turbance was not completely rejected by the controller because of measurement noise and stiction-like effects in the die gaps.

The purpose of the next eloeed loop experiment was to test the closed loop performance with the controller designed above $(k=$

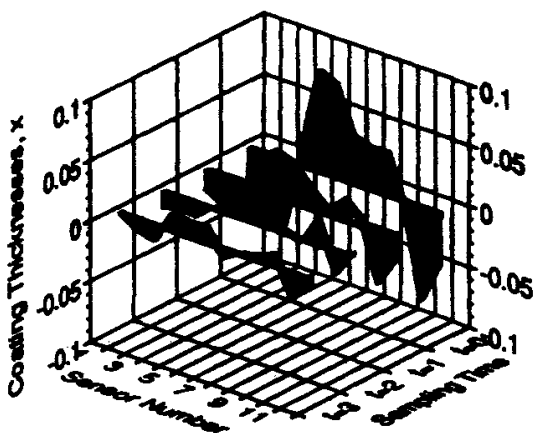

Figure 8: Cloeed loop reeponse for $k=0.05$.

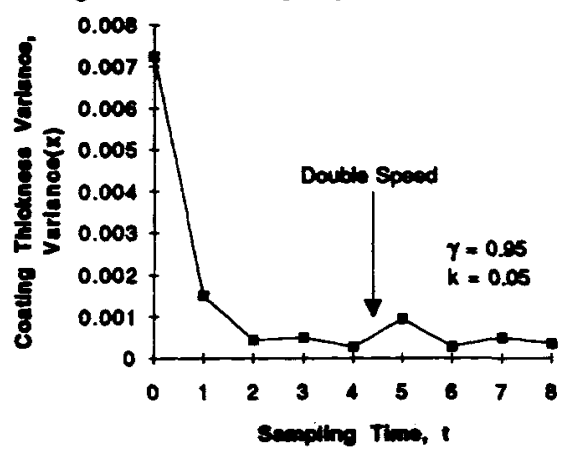

Figure 9: Closed loop response for two disturbances.

$0.05, \gamma=0.95$ ). Fig. 9 shows the closed loop response (the variance of the coating thicknesses) with the designed controller to two types of disturbances. The first disturbance was caused by a roller that had a larger radius for the intermediate sensor positions than for the edge positions - this disturbance was rejected within 2 sampling times as shown in Fig. 9. The second disturbance was caused by ramping the roller speed and liquid flow rates (in a constant ratio) to double their values between the fourth and fift sampling instances. The nominal gap width was kept at a constant value. We from Fig. 9 that changing the roller speed and liquid flow rates in a constant ratio does not subetantially affect the variance of the coating thicknesses.

\section{Conclusions}

A model predictive control algorithm was presented which rejects slow disturbances in coating thicknesses. The control algorithm has one tuning parameter $\gamma$, which trades robustness to model error and insensitivity to measurement noise with speed of response. The simplest yet effective constraint-handling method involved scaling the control action by a scalar which was just large enough to make the control action feasible.

Actuator constraints, measurement noise, and model uncertainty are investigated to determine which of these limit the achievable closed loop performance. Knowledge of how these limitations affect the performance suggested how to modify the plant to improve the uniformity of the coating process. Also we found that the controlle was very forgiving of a poor gain estimate. This means that identification runs are not needed to design the controller when a reasonable estimate of the plant gain is available.

The theory developed throughout the paper was applied to a pilot plant coating process at the Avery/Dennison Research Center in Pasadena. The effect of interactions on the closed loop performance was shown to be negligible for this pilot plant. The measurement noise and the actuator constraints were shown to have the largest effect on closed loop performance.

\section{Acknowledgement}

Piet van Emmerik and Daniel Logue of the Avery/Dennison Re search Center in Pasaden are acknowledged for encourasement and financial support of this work.

References

[1]K. J. Aström and B. Wittenmark. Computer Coatrolled Systems Theory and Design. 1984.

[2] P. J. Campo. PhD thesis, Caltech, 1990.

[3] Manfred Morari et al. Model Predictive Control. 1992.

4] R. D. Braatz et al. Technical report, Caltech, 1991.

[5]S. Skogestad et al. IEEE Auto. Control, AC-33:1092-1105, 1988. [6] Lennart Ljung. System identification: theory for the ser. 1987. 


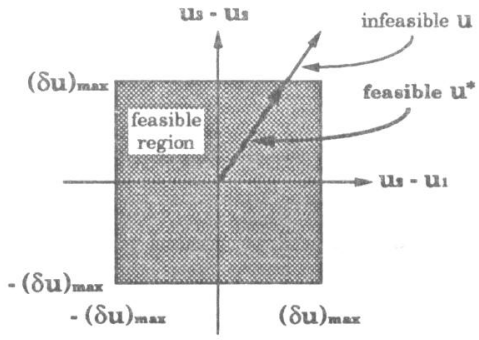

Figure 2: Projection of an infeasible control action to the feasible space. 